\title{
Primera descripción de la composición y distribución de crustáceos decápodos en los canales de la Patagonia central (Chile): observaciones in situ obtenidos mediante un ROV en las aguas interiores de la Reserva Nacional Katalalixar
}

\author{
MATTHIAS GORNY ${ }^{1} \&$ RAÚL PEREDA ${ }^{2}$ \\ 1. https://orcid.org/0000-0002-4445-4499, \ mgorny@oceana.org \\ 2. https://orcid.org/0000-0001-7258-6133
}

\section{OPEN ACCESS}

Recibido:

20/12/2021

Revisado:

22/12/2021

Aceptado:

$23 / 12 / 2021$

Publicado en línea:

$31 / 12 / 2021$

Editor invitado:

Dr. Américo Montiel San Martín

ISSN 0718-686X

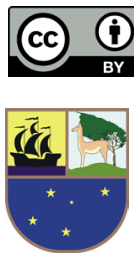

\section{RESUMEN}

Entre 2008 y 2019 se realizó un estudio sistemático de la fauna bentónica dentro de la Reserva Nacional Katalalixar (RNK), ubicada en la Patagonia central, al sur del Golfo de Penas y entre los Campus de Hielo Norte y Sur, con el objetivo de determinar la composición de especies de crustáceos decápodos presentes en esta área geográfica. Los muestreos se llevaron a cabo mediante filmaciones submarinas con un robot submarino (ROV). En total, se identificaron 17 especies de crustáceos decápodos, representando los infraórdenes Caridea (1 especie), Anomura ( 7 especies) y Brachyura ( 9 especies), y distribuidos en 12 familias. Los decápados se encontraron con alta frecuencia, presentes en $80 \%$ de los muestreos realizados con filmaciones entre $240 \mathrm{~m}$ por $220 \mathrm{~m}$. de profundidad. Todas las especies encontradas han sido descritas para la Patagonia Chilena. Sin embargo, con la presencia en el canal Adalberto se extendió el rango de la distribución geográfica hasta el sur de la Península Taitao $\left(48^{\circ} \mathrm{S}\right.$ ) para las jaibas Pisoides edwardsi Nicolet 1894 y Pinnixia bahamondei Garth 1957. Las familias Majidae (Brachyura) y Paguridae (Anomura) eran las más diversas, con 3 especies cada una y el langostino de los canales Munida gregaría Fabricius, 1793 y el camarón Campylonotus vagans Bate, 1888, eran las especies más frecuentes, ambas con un rango amplio de distribución batimétrica de 20 a 100 m y 20 a 120 m de profundidad, respectivamente. Con la presencia de $25 \%$ de las especies descritos para la Patagonia, la fauna de los decápodos de la Reserva Nacional Katalalixar es altamente representativa para la región de los fiordos y canales chilenos, además se encontraron dos especies distribuidas hasta la Antártica. Considerando los pocos estudios del bento de las aguas interiores de la zona central de la Patagonia, los resultados obtenidos en la Reserva Nacional Katalalixar representan un aporte importante al conocimiento de la fauna carcinóloga de Chile.

Palabras claves: Decápodos, Patagonia, Katalalixar, filmaciones submarinas. 


\section{First description of the composition and distribution of decapod crustaceans in channels of central Patagonia (Chile): in situ observations made with a ROV in the inner waters of the Katalalixar Natural Reserve}

\footnotetext{
CÓMO CITAR:

Gorny \& Pereda (2021).

Primera descripción de la composición y distribución de crustáceos decápodos en los canales de la Patagonia Central (Chile): observaciones in situ obtenidos mediante un ROV en las aguas interiores de la Reserva Nacional Katalalixar https://doi.org/10.22352/ AIP202149021

Contribución de los autores:

MG: Muestreos principales con ROV, asistencia en la identificación de especies, elaboración de datos y redacción principal del texto.

RP: Muestreo adicionales, asistencia en la elaboración de datos y redacción del texto.

Declaración de interéses:

Los autores declaran no tener conflicto de intereses.

Financiamiento: Sin financiamiento.
}

\section{ABSTRACT}

Between 2008 and 2019 a systematic study of the benthic fauna was conducted in the Natural Reserve Katalalixar (KNR), located south of the Penas Gulf and between the North and South Patagonian Icefield located in Central Patagonia, in order to determine the composition of decapod crustacean species present in this sparsely investigated geographic area. Registers of decapods were obtained by underwater video with a remotely operated vehicle (ROV). A total of 17 species of decapods was identified, belonging to 12 families, and representing the infraorders Caridea (1 species), Anomura (7 species) and Brachyura (9 species). Decapods were frequent, occurring in $80 \%$ of the stations between 5 and $220 \mathrm{~m}$, of visual documentations by ROV. All identified species were registered in Chilean Patagonia before. However, the presence of the crabs Pisoides edwardsi Nicolet 1894 and Pinnixia bahamondei Garth 1957 in the Adalberto channel extended the known geographical distribution range towards south of the Taitao Peninsula $\left(48^{\circ} \mathrm{S}\right.$ ). The families Majidae (Brachyura) and Paguridae (Anomura) were the most divers ones, represented by 3 species each and the squat lobster Munida gregaría and the shrimp Campylonotus vagans were the most frequent species, with a wide bathymetric range, distributed between 20 and 100 and 20 to $120 \mathrm{~m}$, respectively. The decapod fauna of the KNR is highly representative, comprising $25 \%$ of the species described for the Chilean fjord and channel region, and 2 species distributed even around Antarctica. The results obtained in the KNR signify an important contribution to the knowledge of the fauna of crustaceans of Chile, considering that only few studies have been carried out in Central Patagonia before,

Keywords: Decapods, Patagonia, Katalalixar, underwater video.

\section{INTRODUCCIÓN}

La Reserva Nacional Katalalixar (RNK) se encuentra en el centro de la Patagonia occidental (extremo austral de Chile) dentro de la zona de los canales y fiordos de la Región de Aysén. Esta zona que ha sido moldeada por épocas glaciares recientes (Soto, 2009) y la cual forma gran parte de la ecorregión marina Kawésqar (Rovira \& Herreros, 2016). 
Su ubicación y la presencia de los campos de hielo, además de la cercanía de la Patagonia con la Antártica resultan en un clima caracterizado por elevadas precipitaciones (Soto et al. 2013) y fuertes vientos catabáticos que bajan con frecuencia desde la cordillera hacia el mar (Calvete, 2002; Sievers \& Silva, 2008).

Debido a las fuertes precipitaciones y deshielos, grandes descargas de agua dulce se desplazan rápidamente desde las montañas por medio de ríos y riachuelos hacia el mar, modelando las condiciones oceanográficas de toda la región de los fiordos y canales patagónicos (Iriarte et al. 2014), esta presencia de capas de agua dulce que se sobreponen a las aguas oceánicas saladas resultan en una estratificación bien marcada, con gradientes de temperatura, salinidad y densidad, entre otros parámetros oceanográficos (Pickard, 1971; Sievers \& Silva, 2008; Iriarte et al. op. cit.).

El conocimiento sobre la composición y distribución de los crustáceos decápodos en los fiordos y canales patagónicos ha sido obtenido de diversas expediciones desde la expedición del Challenger (1873-1883; $\left.40^{\circ}-63^{\circ} \mathrm{S}\right)$, Hamburger Magalhaensische Sammelreise (1892-1893,39 $\left.{ }^{\circ}-55^{\circ} \mathrm{S}\right)$, B.A.N.Z. Antarctic Research Expedition (1929-1931) $41^{\circ}-68^{\circ} \mathrm{S}$ and $159^{\circ}-49^{\circ} \mathrm{E}$, Lund University Chile

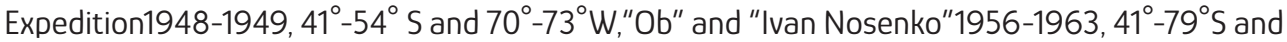
$166^{\circ} \mathrm{E}-189^{\circ} \mathrm{W} 14$, USNS "Eltanin" cruises $1962-1972,33^{\circ}-69^{\circ} \mathrm{S}$ and $74^{\circ}-162^{\circ} \mathrm{W}$ y la expedición internacional de R/V Victor Hensen del 1994, abarcando el área del estrecho de Magallanes hasta el sur del Canal Beagle (cf. Gorny, 1999). Los resultados de todas esas colecciones de crustáceos decápodos en el extremo sur de Chile se encuentran en diversas publicaciones (Retamal, 1973, 1974, 2000; Gorny, 1999; Retamal \& Gorny, 2001; Retamal \& Moyano, 2010). Sin embargo, el único estudio sobre decápodos de la Patagonia central se realizó en el marco de los cruceros CIMAR FIORDOS, pero por gran parte en el área oceánica y al sur de la RNK (Mutschke $\varepsilon$ Gorny, 1999).

El objetivo principal del estudio fue ampliar el conocimiento sobre la fauna carcinológica de las aguas interiores de la Patagonia central, aprovechando los muestreos biológicos y filmaciones submarinas realizados dentro de la RNK entre 2008 y 2019 durante varias expediciones (Gorny et al. 2020).

\section{MATERIAL Y MÉTODOS}

La obtención de muestras correspondientes a las 40 estaciones totales, obtenidas en expediciones realizadas a partir del 2008 y hasta el 2019, por parte de la ONG OCEANA y en conjunto con la Universidad de Magallanes (UMAG) y la Corporación Nacional Forestal Región de Aysén (CONAF).

Las técnicas utilizadas para la obtención de muestras biológicas correspondieron a colecciones no cuantitativas mediante buceo autónomo (4 estaciones) en aguas someras entre 5 y $20 \mathrm{~m}$ de profundidad, las cuales fueron extraídas y fijadas con etanol al $95 \%$ para su posterior traslado y análisis en laboratorio. Por otro lado, se realizaron 32 filmaciones submarinas mediante el uso de un ROV (Remotely Operated Vehicle) entre 10 y 220 m, de la marca Mariscope Meerestechnik, Kiel, Alemania, equipado con una videocámara HD (Panasonic SD 909, resolución 1920×1080, 30 fps), una cámara GoPro Hero (resolución 2704×1520, 60 fps) y dos punteros láser (montados a $10 \mathrm{~cm}$ de distancia entre las cámaras frontales). Los vídeos de 2008-2009 se obtuvieron con un ROV más pequeño y se filmaron en HD ( $1440 \times 1080,30$ fps). Ambos ROVs contaban con luces 
Fig. 1. Ubicación de la Reserva Nacional

Katalalixar en la

Patagonia Chilena

Central (verde) y puntos de filmaciones con ROV. Se indica la riqueza (número de especies por estación) de crustáceos decápodos

Fig. 2. Número de especies acumulado por estación de muestreo.
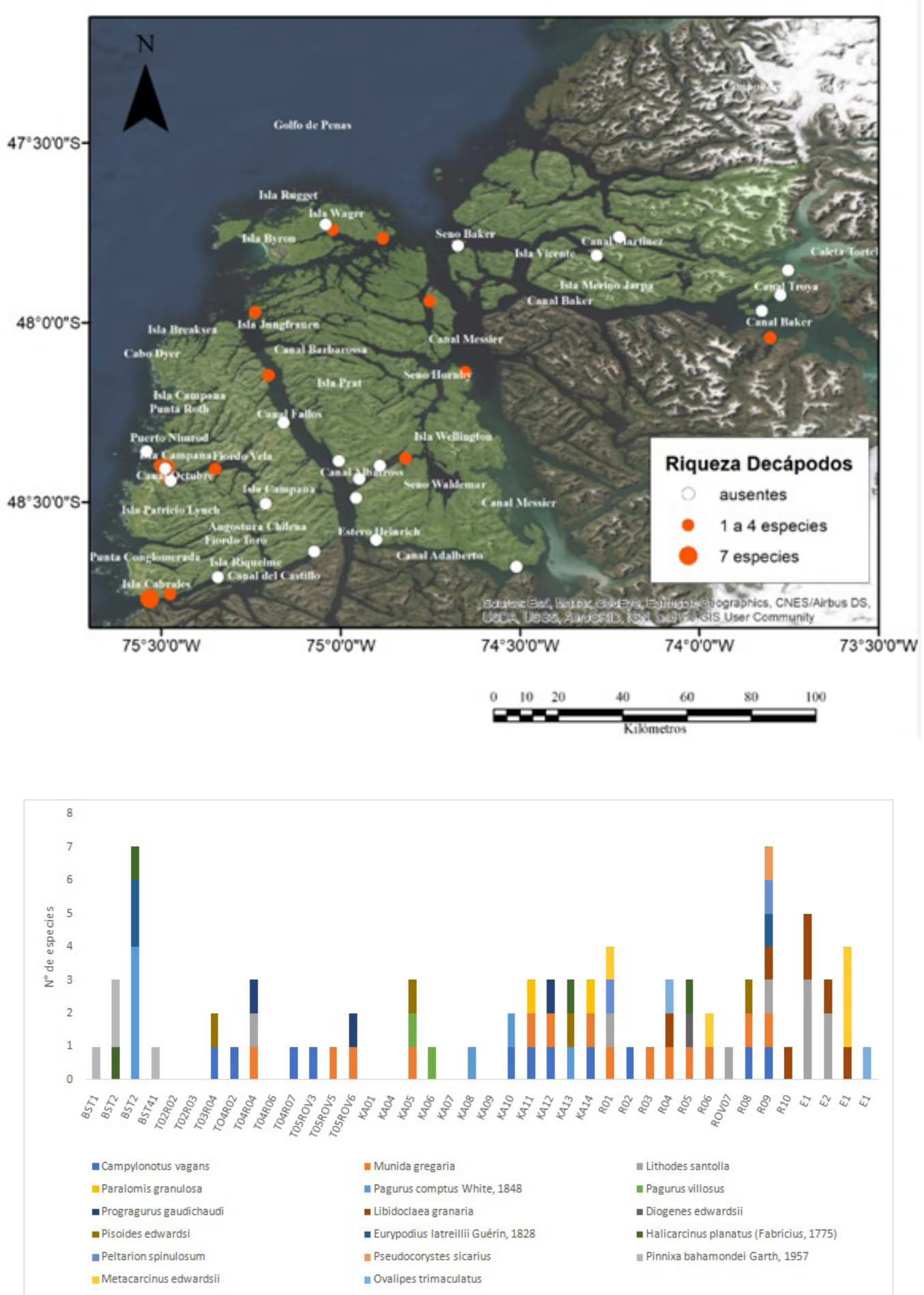
Tabla 1. Lista de estaciones y sus georeferencias.

R: ROV B: Buceo C: Trampa centollera I: Intermareal J: Trampa jaibera.

\begin{tabular}{|c|c|c|c|c|c|}
\hline \multicolumn{2}{|c|}{ Estación } & Año & Prof. (m) & Latitud $\left({ }^{\circ} \mathrm{S}\right)$ & Longitud $\left({ }^{\circ} \mathrm{O}\right)$ \\
\hline $\mathrm{R}$ & T03R04 & 2009 & $90-130$ & $-47,96853$ & $-73,82586$ \\
\hline $\mathrm{R}$ & T04R02 & 2009 & $60-90$ & $-47,81488$ & $-74,28606$ \\
\hline $\mathrm{R}$ & T04R04 & 2009 & $20-30$ & $-48,04307619$ & $-73,80218125$ \\
\hline $\mathrm{R}$ & T04R07 & 2009 & $30-50$ & $-47,85601$ & $-73,75242$ \\
\hline $\mathrm{R}$ & T05ROV3 & 2010 & $20-60$ & $-48,68138$ & $-74,50942$ \\
\hline $\mathrm{R}$ & T05ROV5 & 2010 & 20 & $-47,72799$ & $-75,04128$ \\
\hline $\mathrm{R}$ & T05ROV6 & 2010 & $20-40$ & $-47,74216$ & $-75,02198$ \\
\hline $\mathrm{R}$ & KA05 & 2017 & 30 & $-48,4091$ & $-75,35025$ \\
\hline $\mathrm{R}$ & KA06 & 2017 & 70 & $-48,3986$ & $-75,50439$ \\
\hline $\mathrm{R}$ & KA08 & 2017 & 63 & $-48,7574$ & $-75,4752$ \\
\hline $\mathrm{R}$ & KA10 & 2017 & $13-27$ & $-48,14741$ & $-75,200879$ \\
\hline $\mathrm{R}$ & KA11 & 2017 & $40-50$ & $-47,9716$ & $-75,23873$ \\
\hline $\mathrm{R}$ & KA12 & 2017 & $40-50$ & $-47,7664$ & $-74,88182$ \\
\hline $\mathrm{R}$ & KA13 & 2017 & 27-oct & $-47,94158996$ & $-74,75179296$ \\
\hline $\mathrm{R}$ & KA14 & 2017 & $30-220$ & $-48,13906$ & $-74,65298$ \\
\hline $\mathrm{R}$ & R01 & 2018 & 20 & $-48,50562$ & $-75,20874$ \\
\hline $\mathrm{R}$ & R02 & 2018 & $60-90$ & $-48,4076$ & $-75,4883$ \\
\hline $\mathrm{R}$ & R03 & 2018 & $150-160$ & $-48,4414$ & $-75,4732$ \\
\hline $\mathrm{R}$ & R04 & 2018 & $40-50$ & $-48,4076$ & $-75,4883$ \\
\hline $\mathrm{R}$ & R05 & 2018 & $20-40$ & $-48,4091$ & $-75,3503$ \\
\hline $\mathrm{R}$ & R06 & 2018 & $60-70$ & $-48,3986$ & $-75,5044$ \\
\hline $\mathrm{R}$ & ROV07 & 2018 & $30-80$ & $-48,7104$ & $-75,3418$ \\
\hline $\mathrm{R}$ & R08 & 2018 & $20-50$ & $-48,7574$ & $-75,4752$ \\
\hline $\mathrm{R}$ & R09 & 2018 & $40-60$ & $-48,7702$ & $-75,5324$ \\
\hline $\mathrm{R}$ & R10 & 2018 & 20-145 & $-48,6390$ & $-75,0732$ \\
\hline B & BST1 & 2018 & $5-20$ & $-48,50562$ & $-75,20874$ \\
\hline B & BST2 & 2018 & $5-21$ & $-48,4076$ & $-75,4883$ \\
\hline B & BST3 & 2018 & $5-22$ & $-48,4414$ & $-75,4732$ \\
\hline B & BST4 & 2018 & $5-23$ & $-48,7104$ & $-75,3418$ \\
\hline C & E1 & 2019 & 40 & $-47,500$ & $-74,600$ \\
\hline C & E2 & 2019 & 40 & $-47,500$ & $-75,800$ \\
\hline J & E1 & 2019 & 20 & $-47,500$ & $-74,600$ \\
\hline I & E1 & 2019 & 0 & $-47,400$ & $-75,100$ \\
\hline
\end{tabular}


delanteras y una brújula electrónica y un sensor de profundidad. Estos datos se presentaron como texto de video en el monitor de superficie y se grabaron por separado y en tiempo real en una computadora portátil conectada directamente al control remoto del ROV. Todas las filmaciones se realizaron en forma de transectas, comúnmente perpendicular a la costa, subiendo sobre el fondo desde el punto más profundo hacia la superficie (Gorny et al. 2020). Luego los videos fueron analizados posteriormente en gabinete, usando el programa GOM Player (versión GOM Player Plus 2.3.52.5316). Con el fin de completar el inventario taxonómico se realizaron dos muestreos puntuales mediante una trampa tipo centollera colocada en $40 \mathrm{~m}$, una trampa tipo jaibera en $20 \mathrm{~m}$ y algunos registros de decápodos, colectados en un muestreo manual en la zona intermareal de las islas Wager/Byron. Las posiciones geográficas y el rango de profundidades de las filmaciones con ROV y colección complementaria de decápodos se encuentra en la Tabla 1 y Fig. 1.

\section{RESULTADOS}

Se registró un total de 17 especies de crustáceos decápodos bentónicos en el área de estudio, perteneciente a tres infraordenes y 12 familias de decápodos (Tabla 2 a y b). Las especies se distribuyeron entre el infraoden Caridea con una especie, Anomura con siete y Brachyura con nueve especies. Las familias Majidae (Brachyura) y Paguridae (Anomura) eran las más diversas, con 3 especies cada una. Para la familia de Lithodidae se registraron dos especies (Lithodes santolla y Paralomis granolusa) y una sola especie para las otras familias.

La distribución de este grupo se observó en gran parte de la Reserva Nacional Katalalixar; desde las confluencias de los canales con el océano en el extremo oeste donde destacan los sectores oceánicos del canal Castillo con un mayor número de especies registradas por estación (7 especies.) (Fig. 2) y hasta las aguas interiores, al sur del canal Troya en el extremo sureste de la reserva (1 especie.). Los registros de Pagurus villosos y de Pinnixia bahamondei en el canal Adalberto $\left(48^{\circ} 22^{\prime} S\right.$ ) representan los registros más australes para ambas especies, según los datos indicados por Retamal \& Moyano (2010).

El langostino de los canales Munida gregaria (Galatheidae) (Fig. 3a) con registros en 14 estaciones y el camarón Campylonutus vagans (Campylonotidae) (Fig. 3b) con 11 registros eran las especies más frecuentes. Aunque las filmaciones in situ no eran muestreos cuantitativos, la especie más abundante era M. gregaria con densidades estimadas de hasta más de 40 individuos por metro cuadrado, observadas cerca de la confluencia oceánica del canal del Castillo (Fig. 3a). Otras aglomeraciones de langostinos, pero con densidades menores, se observaron al sur de las islas Byron/Wagner cerca del Golfo de Penas y durante invierno de 2008 al sur del canal Troya.

Los registros de los decápodos se hicieron en profundidades entre 1 y $160 \mathrm{~m}$, la mayoría de las especies se encontró entre 20 y $60 \mathrm{~m}$ (Tabla 3). Un amplio rango de distribución vertical entre 20 a 120 m ocupó el camarón Campylontus vagans y el langostino de los canales Munida gregaria (20 a $100 \mathrm{~m}$ ). El registro de un individuo del centollon Paralomis granulosa en $150 \mathrm{~m}$ en el canal Messier (estación KA01R14) era el más profundo de los decapados observados dentro de la Reserva Nacional Katalalixar. Pinnixa bahamondei (Pinnotheridae) y Halicarcinus planatus (Hymenosomatidae) eran las únicas especies que se encontraron solo en aguas muy someras entre 5 y $40 \mathrm{~m}, \mathrm{H}$. planatus, ha sido registrado con una alta presencia en la zona intermareal del área norte de la reserva nacional, específicamente en islas Byron y Wager ubicadas en el archipiélago de Guayaneco. 
Fig.3a.

Registro de Munida gregaria.

Fig.3b.

Registro de Campylonotus vagans.
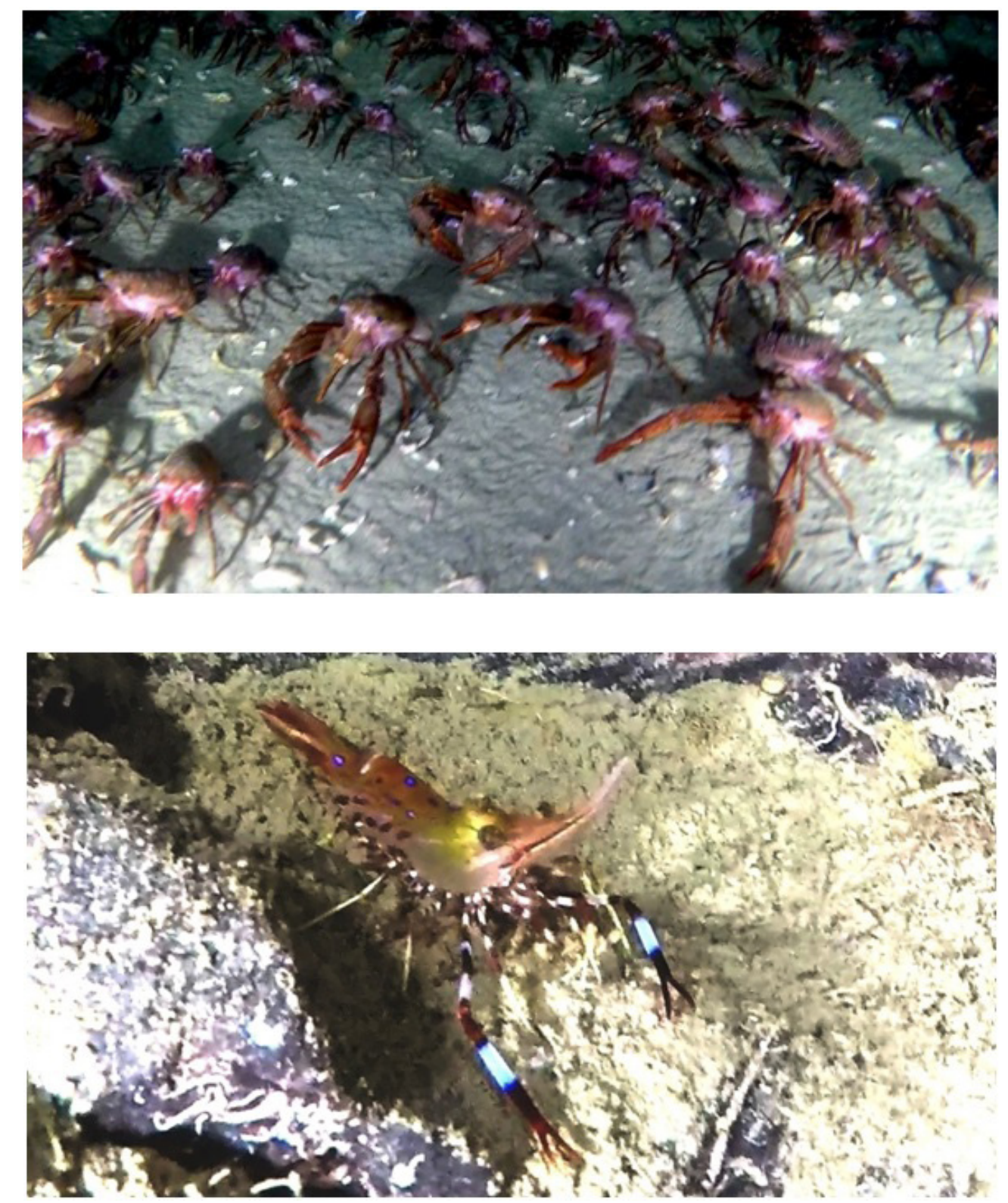

Por otro lado, el número de especies registradas para cada estación, indico que dos de estas estaciones registran siete especies, correspondiendo esto al 11,7\% de las especies registradas, en el $88,2 \%$ restante se registraron un acumulado por estación menor a cinco especies (Fig. 2) Importante es señalar que el mayor registro de especies por estación correspondieron a estaciones de buceo autónomo (1) y filmaciones con ROV (1) realizadas en el 2018 y en la confluencia de los canales con el océano Pacifico. 
Decápodos de la Reserva Nacional Katalalixar

Tabla 2a. Registros de crustáceos decápados en la Reserva Nacional Katalalixar, obtenidos entre 2008 y 2019.

\begin{tabular}{|c|c|c|c|c|c|c|c|c|}
\hline & \multirow{3}{*}{$\begin{array}{c}\text { Caridea } \\
\text { Campylonotidae } \\
\begin{array}{c}\text { Campylonotus } \\
\text { vagans }\end{array} \\
\end{array}$} & \multicolumn{7}{|c|}{ Anomura } \\
\hline & & \multirow{2}{*}{$\begin{array}{c}\text { Galatheidae } \\
\text { Munida } \\
\text { gregaria }\end{array}$} & \multicolumn{2}{|c|}{ Lithodidae } & \multicolumn{3}{|c|}{ Paguridae } & \multirow{2}{*}{$\begin{array}{c}\text { Diogenidae } \\
\text { Diogenes } \\
\text { edwardsii }\end{array}$} \\
\hline & & & $\begin{array}{l}\text { Lithodes } \\
\text { santolla }\end{array}$ & $\begin{array}{l}\text { Paralomis } \\
\text { granulosa }\end{array}$ & $\begin{array}{l}\text { Pagurus } \\
\text { comptus }\end{array}$ & $\begin{array}{l}\text { Pagurus } \\
\text { villosus }\end{array}$ & $\begin{array}{l}\text { Progragurus } \\
\text { gaudichaudi }\end{array}$ & \\
\hline T03R04 & 1 & 0 & 0 & 0 & 0 & 0 & 0 & 0 \\
\hline T04R02 & 1 & 0 & 0 & 0 & 0 & 0 & 0 & 0 \\
\hline T04R04 & 0 & 1 & 1 & 0 & 0 & 0 & 1 & 0 \\
\hline T04R07 & 1 & 0 & 0 & 0 & 0 & 0 & 0 & 0 \\
\hline T05ROV3 & 1 & 0 & 0 & 0 & 0 & 0 & 0 & 0 \\
\hline T05ROV5 & 0 & 1 & 0 & 0 & 0 & 0 & 0 & 0 \\
\hline T05ROV6 & 0 & 1 & 0 & 0 & 0 & 0 & 1 & 0 \\
\hline KA05 & 0 & 1 & 0 & 0 & 0 & 1 & 0 & 0 \\
\hline KA06 & 0 & 0 & 0 & 0 & 0 & 1 & 0 & 0 \\
\hline KA08 & 0 & 0 & 0 & 0 & 1 & 0 & 0 & 0 \\
\hline KA10 & 1 & 0 & 0 & 0 & 1 & 0 & 0 & 0 \\
\hline KA11 & 1 & 1 & 0 & 1 & 0 & 0 & 0 & 0 \\
\hline KA12 & 1 & 1 & 0 & 0 & 0 & 0 & 1 & 0 \\
\hline KA13 & 0 & 0 & 0 & 0 & 1 & 0 & 0 & 0 \\
\hline KA14 & 1 & 1 & 0 & 1 & 0 & 0 & 0 & 0 \\
\hline R01 & 0 & 1 & 1 & 0 & 0 & 0 & 0 & 0 \\
\hline $\mathrm{R} 02$ & 1 & 0 & 0 & 0 & 0 & 0 & 0 & 0 \\
\hline R03 & 0 & 1 & 0 & 0 & 0 & 0 & 0 & 0 \\
\hline R04 & 0 & 1 & 0 & 0 & 0 & 0 & 0 & 0 \\
\hline R05 & 0 & 1 & 0 & 0 & 0 & 0 & 0 & 1 \\
\hline R06 & 0 & 1 & 0 & 0 & 0 & 0 & 0 & 0 \\
\hline ROV07 & 0 & 0 & 1 & 0 & 0 & 0 & 0 & 0 \\
\hline R08 & 1 & 1 & 0 & 0 & 0 & 0 & 0 & 0 \\
\hline R09 & 1 & 1 & 1 & 0 & 0 & 0 & 0 & 0 \\
\hline R10 & 0 & 0 & 0 & 0 & 0 & 0 & 0 & 0 \\
\hline BST1 & 0 & 0 & 0 & 0 & 0 & 0 & 0 & 0 \\
\hline BST2 & 0 & 0 & 0 & 0 & 0 & 0 & 0 & 0 \\
\hline BST3 & 0 & 0 & 0 & 0 & 4 & 0 & 0 & 0 \\
\hline BST4 & 0 & 0 & 0 & 0 & 0 & 0 & 0 & 0 \\
\hline E1 & 0 & 0 & 3 & 0 & 0 & 0 & 0 & 0 \\
\hline E2 & 0 & 0 & 2 & 0 & 0 & 0 & 0 & 0 \\
\hline E1 & 0 & 0 & 0 & 0 & 0 & 0 & 0 & 0 \\
\hline E1 & 0 & 0 & 0 & 0 & 0 & 0 & 0 & 0 \\
\hline
\end{tabular}


Tabla 2b. Registros de crustáceos decápados en la Reserva Nacional Katalalixar, obtenidos entre 2008 y 2019.

\begin{tabular}{|c|c|c|c|c|c|c|c|c|c|}
\hline & & \multicolumn{8}{|c|}{ Brachyura } \\
\hline & & Majidae & & Hymenosomatidae & Atelecyclidae & Corystidae & Pinnotheridae & Cangridae & Ovalipidae \\
\hline & $\begin{array}{l}\text { Libidoclaea } \\
\text { granaria }\end{array}$ & $\begin{array}{l}\text { Pisoides } \\
\text { edwardsi }\end{array}$ & $\begin{array}{l}\text { Eurypodius } \\
\text { latreillii }\end{array}$ & $\begin{array}{l}\text { Halicarcinus } \\
\text { planatus }\end{array}$ & $\begin{array}{l}\text { Peltarion } \\
\text { spinulosum }\end{array}$ & $\begin{array}{l}\text { Pseudocorystes } \\
\text { sicarius }\end{array}$ & $\begin{array}{c}\text { Pinnixa } \\
\text { bahamondei }\end{array}$ & $\begin{array}{l}\text { Metacarcinus } \\
\text { edwardsii }\end{array}$ & $\begin{array}{c}\text { Ovalipes } \\
\text { trimaculatus }\end{array}$ \\
\hline T03R04 & 0 & 1 & 0 & 0 & 0 & 0 & 0 & 0 & 0 \\
\hline TO4R02 & 0 & 0 & 0 & 0 & 0 & 0 & 0 & 0 & 0 \\
\hline T04R04 & 0 & 0 & 0 & 0 & 0 & 0 & 0 & 0 & 0 \\
\hline T04R07 & 0 & 0 & 0 & 0 & 0 & 0 & 0 & 0 & 0 \\
\hline T05ROV3 & 0 & 0 & 0 & 0 & 0 & 0 & 0 & 0 & 0 \\
\hline T05ROV5 & 0 & 0 & 0 & 0 & 0 & 0 & 0 & 0 & 0 \\
\hline T05ROV6 & 0 & 0 & 0 & 0 & 0 & 0 & 0 & 0 & 0 \\
\hline KA05 & 0 & 1 & 0 & 0 & 0 & 0 & 0 & 0 & 0 \\
\hline KA06 & 0 & 0 & 0 & 0 & 0 & 0 & 0 & 0 & 0 \\
\hline KA08 & 0 & 0 & 0 & 0 & 0 & 0 & 0 & 0 & 0 \\
\hline KA10 & 0 & 0 & 0 & 0 & 0 & 0 & 0 & 0 & 0 \\
\hline KA11 & 0 & 0 & 0 & 0 & 0 & 0 & 0 & 0 & 0 \\
\hline KA12 & 0 & 0 & 0 & 0 & 0 & 0 & 0 & 0 & 0 \\
\hline KA13 & 0 & 1 & 0 & 1 & 0 & 0 & 0 & 0 & 0 \\
\hline KA14 & 0 & 0 & 0 & 0 & 0 & 0 & 0 & 0 & 0 \\
\hline R01 & 0 & 0 & 0 & 0 & 1 & 0 & 0 & 1 & 0 \\
\hline $\mathrm{R} 02$ & 0 & 0 & 0 & 0 & 0 & 0 & 0 & 0 & 0 \\
\hline $\mathrm{R} 03$ & 0 & 0 & 0 & 0 & 0 & 0 & 0 & 0 & 0 \\
\hline R04 & 1 & 0 & 0 & 0 & 0 & 0 & 0 & 0 & 1 \\
\hline R05 & 0 & 0 & 0 & 1 & 0 & 0 & 0 & 0 & 0 \\
\hline R06 & 0 & 0 & 0 & 0 & 0 & 0 & 0 & 1 & 0 \\
\hline ROV07 & 0 & 0 & 0 & 0 & 0 & 0 & 0 & 0 & 0 \\
\hline R08 & 0 & 1 & 0 & 0 & 0 & 0 & 0 & 0 & 0 \\
\hline R09 & 1 & 0 & 1 & 0 & 1 & 1 & 0 & 0 & 0 \\
\hline R10 & 1 & 0 & 0 & 0 & 0 & 0 & 0 & 0 & 0 \\
\hline BST1 & 0 & 0 & 0 & 0 & 0 & 0 & 1 & 0 & 0 \\
\hline BST2 & 0 & 0 & 0 & 1 & 0 & 0 & 2 & 0 & 0 \\
\hline BST3 & 0 & 0 & 2 & 1 & 0 & 0 & 0 & 0 & 0 \\
\hline BST4 & 0 & 0 & 0 & 0 & 0 & 0 & 1 & 0 & 0 \\
\hline E1 & 2 & 0 & 0 & 0 & 0 & 0 & 0 & 0 & 0 \\
\hline E2 & 1 & 0 & 0 & 0 & 0 & 0 & 0 & 0 & 0 \\
\hline $\mathrm{E} 1$ & 1 & 0 & 0 & 0 & 0 & 0 & 0 & 3 & 0 \\
\hline E1 & 0 & 0 & 0 & 0 & 0 & 0 & 0 & 0 & 1 \\
\hline
\end{tabular}


Fig. 4. Distribución geográfica de los decápodos registrados en la RNK entre el norte de Chile y la Antártica. CN - ChileNorte, CC Chile Central, CH Ecoregion Chiloense-Taitao,

KA Ecorregión Kawésqar, MA Ecorregión Magallanes, TF Tierra del Fuego, SA Islas Subantárticas, AN Antártica

(islas). Las ecorregiones corresponden a la clasificación el Ministerio de Media Ambiente de Chile (Rovira \& Herreros 2016). Se indica con " $x$ " la extensión del registro greografico según los resultados del estudio presente. Fuentes: 1 Retamal \& Moyano, 2010, 2 Gorny, 1999, 3 WoRMS (2021) http://www. marinespecies.org/aphia. php?p=taxdetails\&id $=445026$

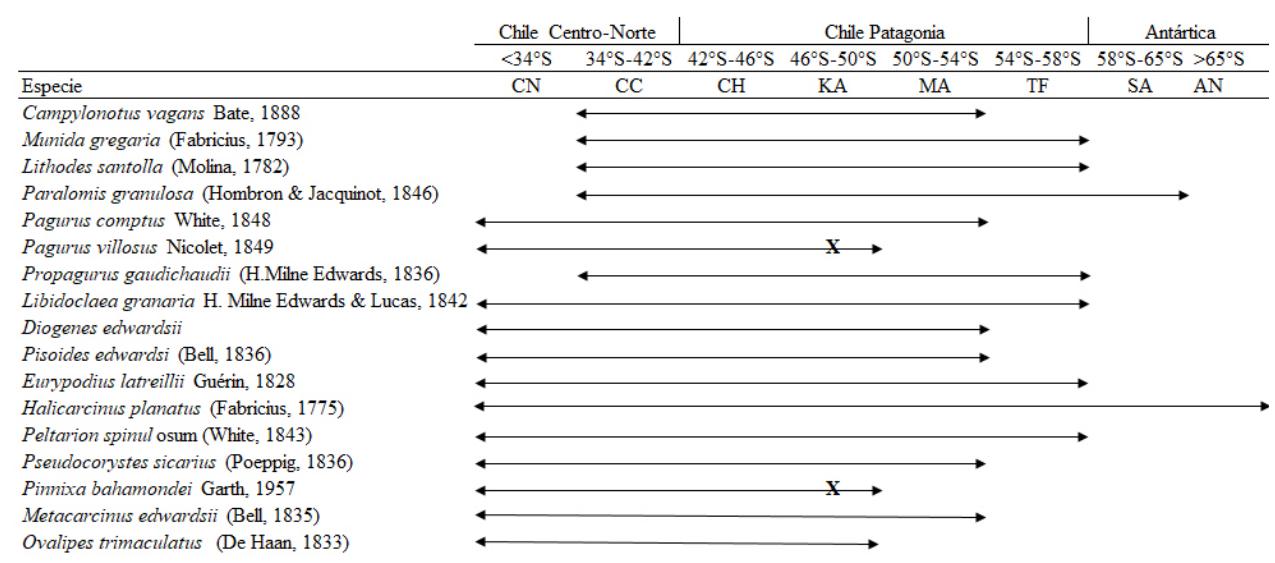

Tabla 3. Rangos de profundidad por especie.

Rangos de Profundidad (m)

\begin{tabular}{|c|c|c|c|c|c|c|c|c|}
\hline Especie & $0-20$ & $20-40$ & $40-60$ & $60-80$ & $80-100$ & $100-120$ & $120-140$ & $140-160$ \\
\hline Pagurus comptus & $x$ & $x$ & & $x$ & & & & \\
\hline Pinnixa bahamondei & $x$ & & & & & & & \\
\hline Campylonotus vagans & & $x$ & $x$ & $x$ & $x$ & $x$ & & \\
\hline Diogenes edwardsii & & $x$ & & & & & & \\
\hline Eurypodius latreillii & & $x$ & $x$ & & & & & \\
\hline Halicarcinus planatus & $x$ & $x$ & & & & & & \\
\hline Libidoclaea granaria & & & $x$ & & & & & \\
\hline Lithodes santolla & $x$ & $x$ & $x$ & $x$ & & & & \\
\hline Metacarcinus edwardsii & $x$ & & & $x$ & & & & \\
\hline Munida gregaria & $x$ & $x$ & $x$ & $x$ & $x$ & & & \\
\hline Ovalipes trimaculatus & & & $x$ & & & & & \\
\hline Pagurus villosus & & $x$ & $x$ & $x$ & & & & \\
\hline Paralomis granulosa & & & $x$ & & & & & $x$ \\
\hline Peltarion spinulosum & $x$ & $x$ & $x$ & & & & & \\
\hline Pisoides edwardsi & & $x$ & $x$ & & & & & \\
\hline Progragurus gaudichaudi & & $x$ & & & & & & \\
\hline Hemigrapsus crenulatus & $x$ & & & & & & & \\
\hline Pseudocorystes sicarius & & & $x$ & & & & & \\
\hline
\end{tabular}




\section{DISCUSIÓN}

Todas las especies encontrados en la RNK han sido registrados previamente en la región de los fiordos y canales de la Patagonia chilena. No obstante, para la Patagonia central la presencia de 17 especies significo un aumento considerable, considerando que solo ocho especies, habían sido registradas previamente frente a los campos de hielo Sur y entre $47^{\circ} \mathrm{S}$ y $51^{\circ} \mathrm{S}$ (Mutschke, 2006). Otros reportes vecinos son proporcionados por Mutschke $\varepsilon$ Gorny (1999), ellos reportan 13 especies de decápodos en aguas oceánicas, frente a las islas de la Patagonia central. Sin embargo, todos los registros anteriores al presente estudio excluyeron las áreas someras.

Principalmente, la fauna de decápodos en el sublitoral rocoso de menos de $240 \mathrm{~m}$ se caracteriza por la presencia de decápodos ermitaños (Paguridae) y una alta diversidad de especies del infraorden Brachyura (cf. Tab.1). No obstante, es interesante que registros de larvas de decápodos en el área de la RNK (Mujica, 2003) coinciden con las especies registrados visualmente o presentes en los muestreos biológicos, indicando que todos los decápodos de los canales interiores se reproducen en el maritorio de la RNK.

La escasa presencia de las especies del infraorden Caridea, representada solo por Campylonutos vagans (Bate, 1888), se podría explicar debido a que los miembros de este infraorden se distribuyen en el extremo sur del Pacifico a profundidades mayores de $250 \mathrm{~m}$ (Gorny, 1999). Además, los registros reportados por Mutschke $\&$ Gorny (1999) y Mutschke (2006) se obtuvieren con redes de arrastre en el fondo de los canales y fiordos, excluyendo los fondos rocosos en las orillas de los fiordos, los cuales fueron filmados en el estudio presente.

El número de especies registradas por cada estación fue altamente variable. En dos estaciones, ubicadas en la confluencia de los canales del Octubre y del Casillo con el océano Pacifico, se registraron 7 especies (Fig. 1). Lo cual corresponde al 11,7\% de todas las especies registradas y al $88,2 \%$ restante se registraron un acumulado por estación menor a cinco especies (Fig. 2).

Desde el punto de vista batimétrico las especies de decápodos se distribuyeron desde la superficie incluyendo el intermareal hasta profundidades cercanas a los $160 \mathrm{~m}$, cuatro de las especies presentan un rango de profundidad por sobre los $100 \mathrm{~m}$ Campylonotus vagans (Bate, 1888), Libidoclaea granaria (H. Milne Edwards y Lucas, 1843), Munida gregaria (Fabricius, 1793) y Paralomis granulosa (Jacquinot, 1847) correspondiendo esto al 9,7\% de las especies registradas. El número restante de $80,3 \%$ de las especies se distribuyó en profundidades menores a los $100 \mathrm{~m}$. Es importante señalar que de este último porcentaje el $58,7 \%$ se encuentra en un rango de profundidad entre superficie y los $60 \mathrm{~m}$ de profundidad.

Datos sobre la composición y la distribución geográfica de especies hoy en día son de alta importancia, considerando que ya se observan cambios en la diversidad y composición de especies terrestres y marinas bajo los efectos del cambio climático (IPCC, 2021). Por otro lado, considerando la representatividad como un factor importante para la conservación de la biodiversidad y de los recursos naturales, los decápodos de la RNK representan especies distribuidos en todo Chile, 
desde el norte de hasta el extremo sur (Fig.4). Todas las especies registradas dentro de la RNK se distribuyen hasta el norte de Chile, mientras que para 11 de ellas el límite sur de su distribución se extiende más al sur del Estrecho de Magallanes.

Se espera que este trabajo no solo significa un aporte al conocimiento de la fauna carcinóloga de Chile, si no también un aporte al conocimiento sobre los ecosistemas de la región de los fiordos y canales australes, para contribuir el futuro manejo y la conservación de este grupo de invertebrados, cuales son de vital importancia para el funcionamiento de los ecosistemas presentes dentro de los maritorios en áreas Silvestres Protegidas de la Patagonia Chilena.

\section{AGRADECIMIENTOS}

Este trabajo se dedica en memoria a mi querido colega Marco Antonia Rivas Q.D.E.P., eximio carcinólogo quien nos dejó una extensa contribución al conocimiento de los decápodos de Chile, a Oceana, quienes han financiado las expediciones para generar los registros presentados en este trabajo y a la Corporación Nacional Forestal (CONAF) por el apoyo tanto administrativo así como también del equipo de guarda parques y profesionales del Departamento de Áreas Silvestres Protegidas de la Región de Aysén.

Finalmente, un agradecimiento especial al Dr. Américo Montiel por su análisis y decápodos colectados en sus muestreos de buceo y también para Germán Zapata-Hernández por identificar los decápodos en las imágenes del ROV.

\section{LITERATURA CITADA}

Brattström H. \& Johanssen A. (1983). Ecological and regional zoogeography of the marine benthic fauna of Chile. Report No 49 of the Lund University Chile Expedition 1948-49. Sarsia 68, 289-339.

Calvete C. (2002). Características oceanográficas físicas y químicas de canales australes chilenos entre el golfo de Penas y el estrecho de Magallanes (Crucero CIMAR 2 Fiordos). Ciencia y Tecnología del Mar, 22(1): 23-88.

Gorny, M. (1999). On the biogeography and ecology of the Southern Ocean decapod fauna. Scientia Marina. 63 (Supl. 1): 367-382.

Gorny. M., Montiel. A, Zapata. G. \& Pereda. R. (2020). En: Oceanografia: Desvelando la belleza, los misterios y los desafíos del mar, cap 3: Las comunidades marinas bentónicas de la reserva nacional Katalalixar (Chile), Artemis, Brasil. Häusserman V. \& G. Förstera. (2009). Fauna Marina Bentónica de la Patagonia Chilena. Santiago Nature in Focus, 1000 pp.

Iriarte J.L., Pantoja S., Iriarte L., Daneri G. (2014). Oceanographic processes in Chilean fjords of Patagonia: from small to large scale studies. Progress in Oceanography 129, 1-7.

IPCC. 2021. Climate Change 2021: The Physical Science Basis, the Working Group I contribution to the Sixth Assessment Report of the Intergovernmental Panel on Climate Change. Masson-Delmotte, V, Zhai, P, Pirani, A, Connors, SL, Péan, C, Berger, S, Caud, N, Chen, Y, Goldfarb, L, Gomis, MI, Huang, M, Leitzell, K, Lonnoy, E, Matthews, JBR, Maycock, TK, Waterfield, T, Yelek çi, O, Yu, R \& Zhou B (eds.). Cambridge University Press. In Press.

Mujica, A. (2003). Larvas de crustáceos decápodos de la zona de Aysén. Ciencia y Tecnología del Mar, 26(2): 109-121. Mutschke, E. \& M. Gorny, M. (1999). The benthic decapod fauna in the channels and fjords along the South Patagonian Icefield, Southern Chile. Scientia Marina. 63 (Sup/ 1): 315-319.

Mutschke, E. (2006). Biodiversidad y estructura de la comunidad macrobentónica en canales y fiordos australes. En: Avances en el conocimiento oceanográfico de las aguas interiores chilenas, Puerto Montt a cabo de Hornos. N. Silva \& S. Palma (eds.) Comité Oceanográfico Nacional - Pontificia Universidad Católica de Valparaiso, Valparaiso, pp. 133-14. 
Pickard G.L. (1971). Some physical oceanographic features of inlets of Chile. Journal of Fisheries Research Board of Canada 28, 1.077-1.106.

Retamal, M. (1973). Contribución al conocimiento de los Crustáceos Decápodos de la región Magallánica. I. Gayana Zool. 29: 1-24

Retamal, M. (1974). Contribución al conocimiento de los Crustáceos Decápodos de la región Magallánica. II. Gayana Zool. 31: 1-24

Retamal, M. (2000). CD-ROM. Decápodos de Chile. World Biodiversity Database. ETI-Universidad de Concepción. Springer-Verlag.

Retamal, M. A., \& Moyano, H. I. (2010). Zoogeografía de los crustáceos decápodos chilenos marinos y dulceacuícolas. Latin american journal of aquatic research, 38(3), 302-328.

Retamal, M. (2010). Crustáceos decápodos marinos (Crustacea: Decapoda). Bibliografía sobre biodiversidad acuática de Chile. Comité Oceanográfico Nacional, Valparaiso, 225-23

Rovira, J., \& Herreros, J. (2016). Clasificación de Ecosistemas Marinos Chilenos. Una Propuesta del Departamento de Planificación y Políticas en Biodiversidad. Santiago. Minist. Del. Medio Ambient., 48.

Sievers H. \& Silva N. (2008). Water masses and circulation in austral Chilean channels and fjords. In Silva N. \& S. Palma (eds.). Progress in the oceanographic knowledge of Chilean interior waters, from Puerto Montt to Cape Horn. Comité Oceanográfico Nacional - Pontificia Universidad Católica de Valparaíso, Valparaíso. pp 53-58.

Soto MV. (2009) Geografía de la región de los fiordos chilenos. En Häusserman \& Försterra (Eds) 2009. Fauna Marina bentónica de la Patagonia. 43-52pp

Soto E.H., Baez P., Letelier S., Naretto J.\& A. Rebolledo. (2013). Biotopos Marinos intermareales y sublitorales someros entre canal Trinidad y canal Smyth, XII Región, Chile. Ciencia y Tecnología del Mar 36, 91-103.

WoRMS (2021). World register of marine Species. (www.marinespecies.org/visitada en noviembre 2021).

Apéndice: Afiliación declara por cada uno de los autores

Número
afiliación Nombre de la institución y/o organización Afiliación

1 Oceana INC, Santiago Chile

2 CONAF-Tortel

\begin{tabular}{lc}
\hline \multicolumn{1}{c}{ Autor } & Afiliación \\
\hline Matthias Gorny & 1 \\
Raúl Pereda & 2 \\
\hline
\end{tabular}

